\title{
ENZYKLOPÄDIE DES LODZER GETTOS - EIN UNVOLLENDETES PROJEKT DES GETTO-ARCHIVS VON 1944 ${ }^{1}$
}

Der Gegenstand des vorliegenden Beitrags ist die Enzyklopädie des Lodzer Gettos, ein Text, der einmalig in der Holocaustliteratur ist. Zum besseren Verständnis des Dokuments werden zuerst Hintergründe für seine Entstehung, Zeit und Raum, wo er verfasst wurde, kurz dargestellt.

Es handelt sich um das Lodzer Getto (1940-1944), das im Frühjahr 1940 in der besetzten Stadt Lodz, am 11. April 1940 in Litzmannstadt umbenannt, errichtet wurde. Das Lodzer Getto war einerseits ein abhängiges, deutschen Besatzungsbehörden völlig unterstelltes „Wohngebiet der Juden“, anderseits lebten in dieser Zwangsgemeinschaft fast 200000 Menschen, die größtenteils in ihrer jüdischen Tradition verwurzelt waren, obwohl es unter ihnen auch solche gab, die im Herbst 1941 aus dem sogenannten „Alten Reich“ deportiert wurden und in vielen Fällen mit der deutschen Kultur und Gesellschaft stark assimiliert waren. Die Bewohner des Gettos waren von der übrigen Bevölkerung der Stadt abgegrenzt und von der Außenwelt abgeriegelt. Die Nachbarn des „Jüdischen Wohngebietes“ waren vor allem Volksdeutsche, was die Isolation noch verstärkte. Die Organisationsstruktur des Gettos war dreigeteilt und bestand aus der deutschen Gettoverwaltung, der deutschen Polizeiaufsicht (Gestapo, Kripo, Schupo) und der jüdischen scheinbaren „Selbstverwaltung“. Von jüdischer Seite erfolgte der gesamte Geschäftsverkehr mit den deutschen Behörden über den als Judenältesten eingesetzten Chaim Mordechaj Rumkowski, dem befohlen wurde, durch einen Ordnungsdienst im Getto Polizeigewalt auszuüben. Die jüdische Polizei wurde aber immer häufiger für deutsche Zwecke herangezogen, beispielsweise im September 1942 als die Nazis Kinder, Kranke und ältere Menschen in das Vernichtungslager Kulmhof deportiert hatten. Der Älteste der Juden, auch Präses genannt, stand an der Spitze eines gut ausgebauten Verwaltungsapparates, der aus einem Netz

* Dr. habil. Prof. UŁ, Krystyna Radziszewska, Universität Łódź, Institut für Germanistik, Pomorska 171/173, 90-236 Łódź. E-Mail: krystyna.rad@wp.pl.

${ }^{1}$ Umfangreiche Teile dieses Textes wurden in polnischer Sprache in: Radziszewska K., Wiatr E., Polit M., Walicki J., (red.) Encyklopedia getta. Niedokończony projekt z archiwum getta, Łódź 2014 gedruckt. 
von Abteilungen, Zentralen, Kommissionen und Betrieben bestand. Rumkowski organisierte auch Konfiszierungen und Zwangsarbeit. Er versuchte die Existenz des Gettos durch Arbeit abzusichern, was in der Parole „Unser einziger Weg ist Arbeit" seinen Ausdruck fand. Es herrschte eine Arbeitspflicht, der fast die ganze Bevölkerung im Alter vom 10. bis zum 65. Lebensjahr unterzogen wurde. Tausende von Juden fanden Beschäftigung in über hundert Fabriken, die Ressorts genannt wurden. In den Ressorts wurden Waren sowohl für die Wehrmacht als auch für private deutsche Unternehmen hergestellt. Die Strategie der „Rettung durch die Arbeit" basierte auf der Annahme, dass das Reich die im Getto hergestellten Güter dringend braucht und das Arbeitspotential aus dem Getto nicht vernichten wird. Das Leben unter einem Zwang, in der Isolation wurde über einen längeren Zeitraum von vielen Beamten der jüdischen „Selbstverwaltung“ als stabile Existenzbedingungen betrachtet. Viele Institutionen und Einrichtungen wie Schulen, Altenheime, Krankenhäuser, Häuserverwaltungen, verschiedene Dienstleistungen etc. vermittelten ein trügerisches Bild der Normalität.

Eine der Abteilungen der sogenannten jüdischen „Selbstverwaltung“ war das Archiv, das am 17. November 1940 ins Leben gerufen wurde. Diese Stelle unter der Leitung vom Rechtsanwalt Henryk Neftalin sollte ursprünglich die Dokumentation der aufgelösten und die Archivalien der noch bestehenden Institutionen sammeln und diese vor Vernichtung schützen. Der einzige überlebende Mitarbeiter des Archivs, Bernard Ostrowski, meinte, dass das Archiv die Taten und Leistungen des Ältesten der Juden, Chaim Mordechaj Rumkowski, dokumentieren sollte. Die Mitarbeiter haben sich aber vorgenommen, alles zu beschreiben, was im Getto passierte und Materialien für spätere Generationen zu sammeln (vgl. AŻIH, 301/2841, Bl.1). Sie sammelten ,,soweit es möglich war, alle das Getto betreffenden Dokumente und [...] schrieben Monographien und Reportagen über die verschiedenen Einrichtungen, Abteilungen und Betriebe“ (vgl. „Archiwum“, In: Encyklopedia 2014, S.14)².

Das Hauptprojekt des Archivs war die Chronik des Gettos, die bis auf wenige Ausnahmen jeden Tag vom 12. Januar 1941 bis zum 30. Juli 1944 geschrieben wurde. Die Autoren dieses Textes waren Juden aus Lodz, Prag und Wien. Viele von ihnen waren Journalisten, die sowohl in der polnischen, als auch in der deutschsprachigen Presse vor dem Zweiten Weltkrieg Artikel veröffentlicht hatten. Trotz veränderter Bedingungen im Getto schrieben sie nach Regeln der Pressesprache. Das Textkorpus umfasst über zweitausend Seiten, die bis Ende 1942 in polnischer, dann in deutscher Sprache verfasst wurden. Die Form der Chronik, vor allem der deutschsprachige Teil, ähnelt einer Zeitung mit festen Rubriken. Nicht alle, für die Zeitung typische Sparten sind in der Chronik vorhanden. Die

${ }^{2}$ Die Lemmata werden in der Enzyklopädie in drei Originalsprachen, d. h. Deutsch, Polnisch und Jiddisch verfasst. In diesem Beitrag wird beim Zitieren auf die Ausgabe in polnischer Sprache verwiesen. 
Berichterstattung konzentriert sich auf die jüdische Zwangsgemeinschaft. Die deutschen Besatzer erscheinen sehr selten im Text. Der Inhalt wird durch wiederkehrende Überschriften, in der Pressesprache als so genannte „Stehzeilen“ bezeichnet, wie „Tagesnachrichten“, „Ressortnachrichten“, „Approvisation“, "Sanitätswesen“, „Justizwesen“, „Fürsorgewesen“, „Gerichtswesen“, „Von der Post", „Man hört, man spricht...“, „Finanzwirtschaft", „Landwirtschaft", „Verkehrswesen“, „Aus der Polizeichronik“, „Humor“ und „Kleiner Getto-Spiegel“ angekündigt. Eins der vielen Hindernisse bei der Arbeit der Chronisten, war die Zensur. Bevor ein Text in die Chronik aufgenommen wurde, wurde er durch die Zensurkommission, die ,im Anschluss an das Archiv arbeitete“ überprüft (,Archiwum“, In: Enzyklopedia 2014, S. 15). Obwohl es den Chronisten anfänglich gar nicht, kurz vor der Auflösung des Gettos nur im bescheidenen Umfang möglich war, die Zustände im Getto kritisch darzustellen und zu kommentieren, stellt die Chronik eine Fundgrube von Informationen aus erster Hand dar, die oft in keinen Dokumenten zu finden sind.

Der Gegenstand dieses Beitrags ist aber ein zweites Projekt des Getto-Archivs, d. h. die so genannte Enzyklopädie des Gettos. Dieser Text wurde im Jahre 1944 auch von einem Autorenkollektiv verfasst. Zum Teil waren es dieselben Autoren, die an der Chronik gearbeitet hatten. Der Gedanke, die Gemeinschaft des Gettos in Form einer Enzyklopädie zu erfassen, tauchte bereits im Jahre 1943 auf. Im Dezember 1943 verfasste Oskar Rosenfeld, ein Schriftsteller und Journalist aus Prag, der 1941 ins Lodzer Getto deportiert wurde, einen Text, der als Einleitung zur Enzyklopädie gedacht war. Der Autor nennt darin die Beweggründe und erklärt die Idee dieses Vorhabens. Ihm zufolge brachte das Zusammenleben einer Gruppe von Menschen unter einem äußeren Zwang Formen hervor, „die eben nur auf dem Boden des Gettos möglich waren“ (Rosenfeld 1994, S. 246). Das Getto schuf sich seine Sprache und seine Terminologie, die mit keiner in der Welt verglichen werden konnte. Rosenfeld weist auf die Veränderung aller sozialen, geistigen und ökonomischen Funktionen hin, die die Veränderung der meisten Begriffe zu Folge hatte. Der Autor führt aus: „Begriffe, die bisher überall unter europäischen Menschen ihren eindeutigen Sinn hatten, unterlagen einer völligen Wandlung. Sie mussten sich den Bedingungen anpassen, die mit Getto ihre Geltung bekommen hatten. [...] Der Wandel der Lebensformen erzwang den Wandel der Begriffsformen" (Rosenfeld 1994, S. 246). Alte Begriffe genügten den Ansprüchen der Gettowelt nicht mehr. Es mussten neue Wörter gebildet werden, die alten mussten einen neuen Sinn bekommen. Die Basis dieses Prozesses erweiterte die Dreisprachigkeit des Gettos, d. h. Polnisch, Jiddisch und Deutsch. Unter den Verfassern der Enzyklopädie waren sowohl Juden aus Lodz als auch so genannte Westjuden, die aus Berlin, Wien, Prag, Hamburg, Düsseldorf, Köln, Trier, Frankfurt am Main und anderen kleineren Städten im Herbst 1941 ins Lodzer Getto deportiert wurden. Sie haben die Lemmata in drei Sprachen verfasst; in Deutsch, Polnisch und Jiddisch. Bei der Arbeit an der Enzyklopädie richteten sie sich sicher 
nach Prinzipien, die der alten jüdischen Tradition des Ordnens und Archivierens des Wissens zugrunde lagen. Für die deutschsprachigen Autoren waren große deutsche Enzyklopädien von Friedrich Arnold Brockhaus und Joseph Meyer, die im 19. Jahrhundert auf den Buchmarkt kamen, ein Vorbild. Auf Meyers Konversations-Lexikon beruft sich Oskar Rosenfeld im Lemma „Klepsydra“. In Berlin erschienen zwei deutschsprachige jüdische Enzyklopädien; Jüdisches Lexikon: ein enzyklopädisches Handbuch des jüdischen Wissens und Encyclopedia Judaica; Das Judentum in Geschichte und Gegenwart, mit denen die deutschsprachigen Autoren aus dem „Alten Reich“ vertraut waren. Die Wurzeln für die jüdischen Enzyklopädien sind allerdings in zwei großen Werken des jüdischen Geistes zu finden; in der Mischna und im Talmud. Seit dem 12. Jahrhundert versuchten einige jüdische Gelehrte das Wissen zu sammeln und zu ordnen und eine allgemeine Enzyklopädie, die der jüdischen Thematik gewidmet werden sollte, in Hebräisch zu verfassen. Diese Versuche scheiterten aber meistens am Geldmangel. Ein gelungenes Vorhaben war die englischsprachige The Jewish Encyklopedia, die auf die Initiative des österreichischen jüdischen Journalisten Isidore Singer zurück geht (vgl. Polit 2014, S. XXIII). Einen Erfolg hatte Jewriejskaja Encyktopiedia in Russisch, die 1908-1913 in St. Petersburg erschien. Unter ihren Mitarbeitern waren u.a. Simon Dubnow und Schalom Kamieniecki, der spätere Archivar und Autor der Chronik aus dem Lodzer Getto.

Das Wort „Enzyklopädie” wurde zum ersten Mal 1532 von François Rabelais in Gargantua und Pantagruel, damals noch im Sinne der Gesamtheit der menschlichen Erkenntnis, gebraucht. Die großen und wichtigen Enzyklopädien entstanden in der Aufklärung. Die mit Abstand berühmteste war die französische Encyclopèdie (1751-1772). Im englischsprachigen Raum war es die Encyklopaedia Britannica (1768-1771). Im 19. Jahrhundert haben Funktion und Form populärer Nachschlagewerke erhebliche Veränderungen durchlaufen. Heutzutage versteht man unter Enzyklopädie ein umfangreiches Nachschlagewerk, das entweder das gesamte allgemeine Wissen oder ein erschöpfendes Spektrum an Material über einen spezialistischen Gegenstand versammelt und ordnet. Wenn es um den Inhalt geht, spricht man von einer allgemeinen (universalen) oder einer Fach- oder Spezialenzyklopädie. Die einzelnen Lemmata können alphabetisch oder nach einem System, d. h. systematisch geordnet sein, daher hat man es mit einer alphabetischen oder systematischen Anordnung zu tun.

Im Falle der Enzyklopädie des Gettos hat man es mit einer Spezialenzyklopädie zu tun. Sie bezieht sich zwar nicht auf ein einziges Fachgebiet, betrifft aber nur eine einzige Gemeinschaft „unter einem äußeren Zwang“ (Rosenfeld 1994, S. 246).

Eine Enzyklopädie besteht aus Lemmata, die mit einem Stichwort eingeleitet werden. Das Stichwort signalisiert den Inhalt und führt in den zu beschreibenden Bereich ein. Es könnte sowohl ein Gegenstand, ein Phänomen, eine Tätigkeit, ein abstrakter Begriff oder eine Person sein. Biographische Stichworte beginnen mit 
dem Familiennamen, nicht mit dem Vornamen. Nach diesem Prinzip richten sich die Autoren der Enzyklopädie des Gettos, die eine Reihe von Gettopersönlichkeiten miteinbezogen haben, die „kraft ihres Amtes oder kraft ihrer den Durchschnitt überragenden Begabung (Individualität) Anspruch darauf erheben können, in einer lexikalen Kulturgeschichte des Gettos Litzmannstadt verzeichnet zu sein" (Rosenfeld 1994, S. 248). Nach dem Familiennamen erscheint der Vorname und dann ein eventueller wissenschaftlicher oder aristokratischer Titel, zum Beispiel: „Gutman Izak, Ingenieur“, „Helman Dawid Lajb, Dr. med.“, „Meyer Martin, Baron von“, „Rosenfeld Oskar, Dr. phil.“. Biographische Lemmata werden nach einem ziemlich einheitlichen Schema verfasst. Gewisse Informationen wie Geburtsdatum, Sterbedatum im Falle des Verstorbenen, Stelle oder Stellen im Getto treten in fast allen biographischen Lemmata auf. Bei den prominentesten Persönlichkeiten wie Rumkowska Helena (die Schwägerin des Judenältesten), Rumkowski Józef (Bruder des Judenältesten), Henryk Neftalin (vertrauter Mitarbeiter von Ch. M. Rumkowski) wird ihr Lebenslauf aus der Vorkriegszeit dargestellt.

In der Einleitung von Rosenfeld findet man auch einen kritischen Hinweis auf einige wichtige Personen, die - dem Autor zufolge - keine aktive Rolle im Getto gespielt haben, aber als 'jüdische Prominente' angesprochen werden können“ (Rosenfeld 1994, S. 248). Zu diesen Persönlichkeiten gehörten Leiter von verschiedenen Abteilungen, Ressorts etc., die sich nicht immer eines guten Rufs unter der Gettobevölkerung erfreuten. Auf ihre negative Rolle wird indirekt in den Lemmata hingewiesen.

Die Stichworte in der Enzyklopädie des Gettos bestehen meistens aus einem Wort, seltener aus zwei oder mehr. Diejenigen zur Bezeichnung von Abteilungen, Institutionen sowie Stellen bestehen meistens aus zwei Worten. Es gibt einige Stichworte, die aus mehreren Worten bestehen, wie „Czapka za kapelusz“ (Mütze gegen Hut), „Talerzyki za gałgany“ (Tellerchen gegen Lumpen), „Galanteria zdobnicza w getcie“ (Schmuck im Getto), „Ivan blust szojfer“ (Ivan bläst Horn) und „Glazers Fabrik in Radegast“. In meisten Fällen steht das Substantiv an erster Stelle. Wenn andere Wortarten betont werden, rücken diese an die erste Stelle („Offenes Getto“, „Freiwillige Aussiedlung“, „Elektrische Straßenbahn“). Der Beitrag „Blockieren“ ist der einzige, der mit einem Verb ausgedrückt wird.

In einer alphabetischen Enzyklopädie unterscheidet man inhaltliche Beiträge und Verweise. Dem polnischen Forscher Alfred Mielczarek zufolge gibt es einige Arten von Verweisen; inhaltliche, synonyme und mit austauschbaren Gliedern (Mielczarek 1967, S. 34). Die letztgenannten betreffen hauptsächlich Spezialenzyklopädien, in denen man auf einzelne Glieder von Termini verweist, die aus mehreren Worten bestehen. In der Enzyklopädie des Gettos findet man viele sowohl inhaltliche als auch synonyme Verweise. Inhaltliche Verweise sollen auf Informationen in bestimmten Lemmata verweisen. Die Autoren benutzen solche Worte wie „vide“, „siehe dort", „patrz tamże“ und verweisen damit auf andere Beiträge, beispielsweise: „Uryson Azriel vide Abbruchstelle“, „Szyszkowice vide 
Heimy“, „Rumki siehe Finanzwesen“, „Karo Mosze s. dort Archiwum“, „Ulice przejazdowe vide szmugiel“, „Zamkniete getto vide Druty“. Synonyme Verweise treten dann auf, wenn die dargestellten Gegenstände oder Phänomene unter mehreren Bezeichnungen bekannt sind, z. B. „Eingesiedelten Abteilung siehe Abteilung für Eingesiedelte“, „Dekret siehe Adoption“, „Resort siehe Fabriken“, „A, B, C Rayone siehe Getto A, B, C“, „Hartgeld siehe Münzen“, „Plecy siehe Protekcje“, „Chaimki vide Rumki“, „Jachsentówka vide Szyszkowice“.

In der Enzyklopädie wichtig sind nicht nur die Lemmata, die verfasst werden, sondern auch diejenigen, die fehlen. Dies ist von besonderer Bedeutung in diesen Werken, die unter einem Zwang entstehen. In der Enzyklopädie des Gettos werden viele Phänomene verschwiegen oder nur angedeutet. Im Lemma „Rojtes Hajzel“" (rotes Häuschen) beschreibt Oskar Rosenfeld nur äußerlich den Sitz der Kripo gegenüber der Marienkirche, wovor alle Bewohner des Gettos Angst hatten, als einen einstöckigen Bau ,in schlichtem gotischen Stil aus roten Backsteinziegeln“ („Rojtes Hajzel“, In: Encyklopedia 2014, S. 177). Die Mitarbeiter des Archivs müssen genau von den Morden und Folterungen gewusst haben, weil ihre Arbeitsstelle nur einige Schritte von dem Kripo-Gebäude entfernt war, erwähnten aber diese mit keinem Wort. Angesichts der ständig drohenden Gefahr seitens der deutschen Kriminalpolizei ist diese Vorsicht verständlich.

In den vorhandenen Teilen der Enzyklopädie des Gettos konzentrierten sich die Autoren auf innerjüdische Angelegenheiten. Die deutschen Besatzer erscheinen nur sporadisch, was auch aussagekräftig ist. Im Lemma „Macht“ bezeichnet Bernhard Ostrowski diese als ,Behörden außerhalb des Getto, die über dieses zu verfügen hatten“ („Macht“, In: Encyklopedia 2014, S. 132). Die deutsche Gettoverwaltung wird im Lemma „Ivan blust szojfer“ erwähnt. Diese populäre jüdische Wendung wird in den Fällen angewendet, wenn ,innerjüdische Angelegenheiten von nicht jüdischen Behörden als zuständig erledigt werden. Im Getto wurde der Ausdruck in den Fällen gebraucht, wo die innerjüdischen Differenzen durch Eingreifen der deutschen Gettoverwaltung liquidiert wurden“ („Ivan blust szojfer“, In: Encyklopedia 2014, S. 98). Im Archiv des Ghetto Fighters House in Israel befindet sich eine Liste mit über 1500 Lemmata, die die Mitarbeiter verfassen wollten. In dieser Liste waren auch einige Lemmata vorgesehen, die sich auf die deutschen Beamten beziehen, z. B. „Biebow“, „Bradfisch Dr.“, „Hämmerle“ und „Ribbe“. Vermutlich konnten die Mitarbeiter diese aus Zeitmangel jedoch nicht verfassen.

Die Enzyklopädie des Gettos sollte die Gesamtheit des Wissens über die Gemeinschaft hinter dem Stacheldraht, über ihre Organisation, ihre Institutionen und Menschen darstellen. Dieses Anliegen der Autoren wurde aber nicht vollständig realisiert. Es fehlen viele Informationen. Einer der Gründe waren sicher die begrenzten Informationsquellen. In den meisten Fällen stützten sich die Verfasser darauf, was sie im Getto sahen oder erlebten. Sie schöpften ihr Wissen darüber hinaus aus den Dokumenten, zu denen sie als Archivarbeiter Zugang hatten. Bernhard Ostrowski, der ehemalige Archivar aus dem Getto behauptet, dass die Infor- 
mationsbeschaffung aus den Kanzleien von Rumkowski problematisch war: Der Älteste der Juden hatte zwei Büros im Getto. Eines am Plac Kościelny befasste sich mit internen Fragen, wozu die Archivare Zugang hatten, dagegen die zweite Kanzlei war am Baluter Ring, wo sich die gesamte Korrespondenz mit der deutschen Gettoverwaltung und der Gestapo befand. Sie war für die Archivare verschlossen. Dora Fuchs, die Leiterin des Sekretariats, hatte die Materialien unter strenger Kontrolle, heißt es bei Bernard Ostrowski (AŻIH, 301/2841, B1. 4).

Eine dominierende Stellung bekommen in der Enzyklopädie Wörter für die Sachen des täglichen Gebrauchs. Oskar Rosenfeld erklärt dies mit der Spezifik des Gettos: „Da die Sorge um die kärglichen Produkte für die Mahlzeit alle anderen Funktionen des animalischen Daseins verdrängt, haben die mit dieser Sorge verknüpften Gegenstände die vordringlichste Bedeutung bekommen - mögen sie durch ein jiddisches oder polnisches oder deutsches Wort ausgedrückt werden“" (Rosenfeld 1994, S. 247). Zu diesen Begriffen gehören u.a. „Babka”, „Salatka”, „Salattunke”, „Kleik”, „Kaffeemischung”, „Kaffee heißer”, „Muschelfleisch”, „Chochla”, „Wydzielaczka” etc. Im Verzeichnis der geplanten Lemmata gibt es viel mehr Begriffe des täglichen Gebrauchs. Was sich hinter diesen Begriffen verbirgt, entspricht nicht unseren heutigen Vorstellungen von diesen Produkten und Gegenständen. Das Anliegen der Autoren war, neue, veränderte Bedeutung der Worte darzustellen. Peter Wertheimer erklärt die veränderte Semantik von „Kaffeemischung" auf folgende Weise:

Eine aus dem Kaffeeersatz, welcher zu den Rationen von meist 300 Gramm zugeteilt wurde und zeitweilig auch im Freiverkauf zu 2-, später 3-, Mk. pro Kilo erhältlich war, [...] unter Beimengung von Zucker, eventuell etwas Margarine oder Öl, Marmelade etc. hergestellte Mischung, die den Feinschmeckertitel „Trüffeln“ erhielt und ungekocht gegessen wurde (,Kaffeemischung“, In: Encyklopedia, S. 103).

Des Weiteren stellt der Autor noch andere Möglichkeiten der Zubereitung essbarer Speisen aus Kaffeeersatz, Mehl und Wasser vor: „Zur Zeit grosser Hungersnot wurden gröbere Kombinationen aus dem Kaffeeersatz hergestellt, z. B. $1 / 4 \mathrm{~kg}$ Kaffeeersatz mit einem Löffel Mehl vermengt, mit etwas Wasser zu festem Teig gemischt und auf der Pfanne getrocknet“ („Kaffeemischung“, In: Encyklopedia, S. 104).

Die heutigen Leser konnotieren den polnischen Begriff „Babka“ anders, als seine Bedeutung im Getto war. Der Eintrag hierzu lautet beispielsweise:

BABKA

Gettokuchen, hergestellt aus geriebenen Kartoffeln, unter Beimengung von etwas Mehl / eventuell geriebenen Rettich, Kohlrübe, etc./, Kaffeeersatz, gesüsst mit Sacharin, /auch diverser Geschmack wurde beigegeben/ und im Wasserbad /ala Pudding/ 1 Stunde bis 1,1/2 Stunden gekocht. Zu Zeiten reichlicher Kartoffelzuteilungen beliebter Brotersatz.

In Hungerepochen wurde diese Babka aus Kartoffelschalen oder aus Kaffeeersatz erzeugt („Babka“, In: Encyklopedia, S. 20). 
Diese Speise war im Getto sehr populär. Die Bezeichnung stammt aus der polnischen Sprache, aber das Wort bekommt im Getto eine völlig andere Bedeutung. In der friedlichen Zeit in Polen bedeutete es einen Sandkuchen, gebacken aus Eiern, Zucker, Milch, Mehl und Butter. Da die meisten Zutaten im Getto nicht zu erhalten waren, hatten sich die hungrigen Menschen einen Ersatz für diesen Kuchen ausgedacht. Ähnlich verhielt es sich mit einem anderen typischen Gettoerzeugnis, mit „Salatka“. Peter Wertheimer definiert diese Speise als Getto-Produkt folgendermaßen:

\begin{abstract}
SALATKA
Gemüsesalat, ursprünglich im Freiverkauf erhältlich, aus saisonmässigen Gemüsen gewöhnlich II. Sorte oder sogar Abfällen unter Zusatz von Säure, Zucker und Gewürzen und künstlichen Aromen /Zitronen, Orangenäther etc. von der Abteilung für Molkereierzeugnisse hergestellt/. Vom Jahre 1942 an rationiert, in Rationen von 150 g. bis 600 g. ausgegeben. Meist als Brotaufstrich verwendet. In Zeiten besonderer Knappheit als Suppe gekocht. Die Salatka wurde in den Milchläden ausgegeben („Salatka“, In: Encyklopedia, S. 191).
\end{abstract}

In der knappen Erläuterung des Begriffs findet man zusätzliche Informationen über das Verteilungssystem der Lebensmittel und darüber hinaus über die Ernährungslage im Getto.

Dieser Beitrag veranschaulicht, wie die Redaktionsarbeit aussah. Auf vielen Karteien befinden sich Korrekturen und Ergänzungen, die mit einem Bleistift eingetragen werden. Auf der Karte mit dem Lemma „Salatka“, dessen Autor Peter Wertheimer ist, hatte Oskar Singer seine Ergänzungen über die Verteilung dieses Produkts eingetragen. Singer hat dieses Lemma überarbeitet und mit seinem Namen signiert. Es gibt mehrere Beiträge, die in zwei oder sogar drei Fassungen vorliegen. („Rumkowska Helena“" "Reichsbaluter“, „Rosenfeld Oskar, dr phil.“

Wenn man den Autorenkollektiv verfolgt, fällt einem auf, dass jeweilige Autoren Interesse für bestimmte Themenbereiche hatten. Der Kettenraucher Oskar Singer verfasst für die Getto-Enzyklopädie einige Einträge, in denen er verschiedene Zigarettensorten definiert, die im Getto angeboten wurden. Der Autor erwähnt die jugoslawischen Zigaretten „Rama“, „Bregova“, „Drava“ und den jugoslawischen Tabak „Savski Duvan“. Die belgischen Zigaretten „Sobor“ werden als eine Sorte dargestellt, die nur für „Bevorzugte und die Hochzeitspaare gelegentlich der Trauungszeremonie als Geschenk des Präses“ (vgl. „Sobor“, In: Encyklopedia 2014, S. 196), bestimmt waren. Der Autor verweist indirekt einerseits auf korrupte Verhältnisse anderseits auf die katastrophale Versorgungslage, in der anlässlich solcher besonderen Zeremonie Zigaretten als Rarität empfunden wurden. In einem anderen Eintrag mit der Überschrift „Ballerina“ bemerkt Singer, dass die gleichnamigen deutschen Zigaretten ,,ausschließlich zur Disposition des Präses standen“ („Ballerina“, In: Encyklopedia 2014, S. 21), was ein zusätzlicher Hinweis auf die gesellschaftliche Hierarchie ist. Bei der Zigaretten- sowie Essens- 
zuteilung fällt die soziale Abstufung im Getto auf. Die höher Gestellten aßen und rauchten auf Kosten ihrer jüdischen Mitmenschen.

Ein anderer Mitarbeiter des Archivs, Oskar Rosenfeld, ein Journalist und Schriftsteller, versuchte die Erfahrung des Gettos künstlerisch zu verarbeiten. Er schrieb zahlreiche Rezensionen, notierte diese in sein privates Tagebuch und bearbeitete Lemmata über kulturelle Ereignisse, obwohl, wie er selber in der Einleitung zur Enzyklopädie schrieb, die ,intellektuellen Bedürfnisse sind in einen engen Rahmen gepresst. Für sie bedarf es nur weniger Worte, Begriffe, Wortverbindungen“" (Rosenfeld 1994, S. 247). Aus seiner Feder stammen solche Lemmata wie „Revue“, „Leihbibliotheken sowie „Wissenschaftliche Abteilung“. Viele Lemmata widmete er Malern, Musikern sowie Komponisten.

Die Mitarbeiter des Archivs beschrieben auch ihre Dienststelle sowie ihre verstorbenen und lebenden Kollegen.

Die Autoren einer Enzyklopädie sollen unsichtbar bleiben. Um dieses Ziel zu erreichen, verfassen sie den Text im Passiv. Allerdings gelingt es ihnen selten die volle Objektivität zu bewahren und oft ist ihr Text keine reine Vermittlung von objektiven Information. In jeder Enzyklopädie spürt man den Geist der jeweiligen Epoche und den individuellen Schreibstil des Verfassers. Es stellt sich die Frage, ob es den Autoren aus dem Getto gelang, objektiv und distanziert zu bleiben. Trotz der in der Einleitung von Oskar Rosenfeld formulierten Versicherung, dass „die Redaktion der Enzyklopädie sich bemüht hat, dem Stoff ohne jede Voreingenommenheit gerecht zu werden“ (Rosenfeld 1994, S. 249), fällte sie mehrmals ihre Urteile und formulierte kritische Bemerkungen über Personen, pathologische Erscheinungen sowie das ganze System im Getto, das auf Macht und Dominanz basierte. Oskar Singer schreibt über Dawid Warszawski, Leiter sämtlicher Schneidereinen im Getto und Vertrauensmann von Rumkowski, dass „er seine Betriebe mit harter Hand führt" sowie dass, er „seinen Arbeitern als hartherziger, rücksichtsloser Chef unbeliebt, ja sogar verhasst war“ („Warszawski Dawid“, In: Encyklopedia 2014, S. 227).

Die Autoren der Enzyklopädie nannten sowohl durch die Blume als auch direkt negative Phänomene, d. h. Korruption, Protektion sowie Ausbeutung der armen Juden durch die privilegierte Elite des Gettos. Im Lemma „Czapka za kapelusz“ hießt es, dass es ,in der Umgangssprache des Gettos eine boshafte Bezeichnung für den gegenseitigen Austausch von korrupten Leistungen war, die unter der Beamtenschaft, vor allem aber von einflussreichen Personen praktiziert wurden. [...] Diese Leistungen, für die eine Gegenleistung verpflichtend war, wurden nicht auf Kosten des Leistenden, sondern auf Kosten der anderen oder des Gemeinwohls durchgeführt. [...] Typisch für sie war die Duldung oder das Vertuschen von kleinerem oder größerem Missbrauch“. („Czapka za kapelusz“, In: Encyklopedia 2014, S. 53). In vielen Bereichen waren Freundschaften von Vorteil. Verschiedene Formen der Korruption, u.a. Bestechung, Vetternwirtschaft, Lösegeldzahlungen, Fälle von Brutalität waren an der Tagesordnung. Józef Zel- 
kowicz machte im Lemma über die Fürsorge im Getto darauf aufmerksam, dass diese Institution ,an ein Schiff ohne Steuer erinnerte. Ihre Beamten erledigten die Angelegenheiten nach ihrer Lust und Laune. Dies musste zur Korruption und Protektion im großen Ausmaße führen“ („Opieka“, In: Encyklopedia 2014, S. 151f). Die Protektion wird wie folgt definiert: „Es ist eine Art Verbindungen im Getto, die es ermöglichen, normale oder sogar zusätzliche Unterstützungen auf vereinfachten Wegen zu bekommen (außerhalb der Warteschlange). Im Getto werden solche Verbindungen an jeder Ecke und in jeder Angelegenheit genutzt. Mit der Zeit wurde dies zur Existenzgrundlage im Getto“ („Protekcja“, In: Encyklopädia 2014, S. 166). Ähnliche Bedeutung hatte der Begriff „Plecy“, der Beziehungen zu einflussreichen Personen im Getto bedeutete, die es ermöglichten, Profite aus etwas zu ziehen („Plecy“, In: Encyklopedia 2014, S. 161). Die pathologischen Zustände werden auch im Lemma „Wazelina“ (Vaseline) zum Ausdruck gebracht. Józef Zelkowicz betont, dass sehr häufig an der Spitze der Ämter, Werkstätten, Fabriken und Institutionen Menschen standen, die noch nie etwas mit der gesellschaftlicher Arbeit zu tun hatten und sich schlugen, um einen Nutzen für sich herauszuziehen („Wazelina“, In: Enzyklopädia 2014, S. 228). Oskar Rosenfeld formuliert eine aussagestarke Äußerung über die Protektion im Lemma „Kolejka“ (Warteschlange), aus dem ersichtlich wird, dass „die Protektionskinder“ an den Verteilstellen, wo die Zuteilungen gekauft werden, sofort abgefertigt werden und so den Wartenden die Ware wegnehmen, während sich kleine Leute der ,peinlichen Prozedur des Anstellens in der Warteschlange" unterziehen müssen (vgl. „Kolejka“, In: Enzyklopedia 2014, 112f.). Auf die soziale Ungleichheit und ökonomische Ungerechtigkeit sowie besondere Privilegien einiger Einwohner weist ein unbekannter Autor des Lemmas „Szyszkowice“ hin. Die Bevölkerung nannte so diesen Teil des Gettos, wo „die Sommerhäuser der einflussreichen Personen konzentriert werden“ („Szyszkowice“, In: Encyklopedia 2014, S. 209). Die Bezeichnung wird vom polnischen Wort „szyszka“ abgeleitet, das „ein hohes Tier“ bezeichnet. Für diese Menschen hat sich noch eine andere Bezeichnung „Dygnitarz", d. h. Würdenträger eingebürgert, die eine einflussreiche Person mit einem überdurchschnittlichen Lebensstandart bedeutete (vgl. „Dygnitarz“, In: Encyklopedia 2014, S. 58).

Kritische Wertung der pathologischen Erscheinungen im Getto wundert nicht, weil die Autoren selbst ein Teil dieser leidenden hierarchisch aufgebauten Gemeinschaft waren. Obwohl das Verhältnis der Verfasser zu den dargestellten Inhalten nicht typisch für eine Enzyklopädie ist, wollten sie diese Phänomene, deren Zeugen sie waren, den nächsten Generationen kritisch vermitteln. Diese Sammlung, d.h. die Wahl der Lemmata, die Art und Weise ihrer Redaktion sowie Bewertung von Personen, Tatsachen und Ereignissen verleiht der Enzyklopädie ein ausdruckstarkes, einzigartiges Gesicht. Vielleicht hatten die Autoren Bedenken, dass die zukünftigen Forscher und Leser, die sich mit der Problematik des Lodzer Gettos befassen und offizielle Dokumente auswerten werden, keinen Einblick in 
die tatsächliche Situation der Menschen im Getto bekommen werden. Oskar Rosenfeld schreibt Folgendes in der Einleitung zur Enzyklopädie über die Rolle des geschriebenen Wortes, die wie eine Botschaft klingt: „In einer späteren Epoche, in derjenigen, die der Erforschung des Gettos angehört, wird solch eine Sammlung, solche eine Enzyklopädie dort Aufklärungen geben können, wo die bloße Schilderung der Zustände nicht ausreicht. Das Wort, die Sprache ist in der Geschichte der Menschheit - die Wissenschaft hat diese These längst bestätigt - ein zuverlässiger Zeuge und Wahrheitsquell als andere, materielle Denkmale" (Rosenfeld 1994, S. 248). Diese visionäre Formulierung eines Getto-Gefangenen und seine Annahme, dass eine „Sammlung der Getto-Begriffe und der Getto-Persönlichkeiten [...] zweifellos über den aktuellen Anlass hinaus Wert haben [wird]", (Rosenfeld 1994, S. 248) wird immer wieder aufs Neue in zahlreichen Veröffentlichungen über das Lodzer Getto bestätigt, in denen sich die Forscher auf die Enzyklopädie berufen, obwohl diese, Rosenfeld zufolge, „keinen Anspruch auf Vollständigkeit und eindeutige Erklärung des Wortsinnes [erhebt]“ (Rosenfeld 1994, S. 248). Die Autoren glaubten aber dem angestrebten Ziel nahe gekommen zu sein. Ihre wertvolle und mutige Arbeit wurde im Sommer 1944 brutal unterbrochen. Im August 1944 wurde das Lodzer Getto aufgelöst und die Archivare wurden nach Auschwitz deportiert. Die Manuskripte u.a. mit den Karteien der Enzyklopädie wurden von dem Briefträger aus dem Getto, Nachman Zonabend gerettet. Heutzutage, wenn die Forschung neben den offiziellen Dokumenten immer mehr Aufmerksamkeit solchen Zeugnissen widmet, in denen sowohl individuelle als auch kollektive Deutungen und Wertungen zu Wort kommen, ist die Bedeutung des Textes der Chronisten aus dem Getto nicht zu unterschätzen. Siebzig Jahre nach dem Verfassen der Enzyklopädie, deren Original im Lodzer Staatsarchiv, im Archiv des Jüdischen Historischen Instituts und im Archiv Ghetto Fighters House zerstreut war, wurde sie von den Wissenschaftlern der Lodzer Universität den Lesern und Forschern zugänglich gemacht.

\section{LITERATURVERZEICHNIS}

Mielczarek A. (1967), Podstawowe wiadomości o encyklopedii. Warszawa.

Polit M. (2014), Encyklopedie żydowskie. Zarys. In: Radziszewska K., Wiatr E., Polit M., Walicki J., (red.) Encyklopedia getta. Niedokończony projekt z archiwum getta, Łódź, S. XXI-XXV.

Radziszewska K. (2011), Flaschenpost aus der Hölle. Texte aus dem Lodzer Getto. Frankfurt am Main u.a.

Radziszewska K., Wiatr E., Polit M., Walicki J., (Hrsg.) (2014), Encyklopedia getta. Niedokończony projekt $z$ archiwum getta, Łódź, S. XIII-XIX.

Ringelblum E. (1955), Notatki. In: Biuletyn Żydowskiego Instytutu Historycznego, Bd. 15-16. S. 271.

Rosenfarb Ch. (1991), Simcha Bunem Szajewicz, In: Di goldene kejt, Bd. 131, S. 9-24.

Rosenfeld O. (1994), Wozu noch Welt. Aufzeichnungen aus dem Getto Lodz, hrsg. von Hanno Loewy, Frankfurt am Main. 


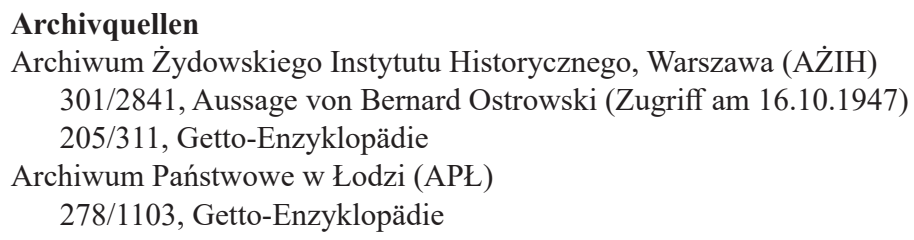

Krystyna Radziszewska

\title{
THE ENCYCLOPEDIA OF THE GHETTO LODZ - AN UNFINISHED PROJECT OF THE ARCHIVE FROM GHETTO 1944
}

\author{
(Summary)
}

The article discusses "The Encyclopedia of the Ghetto". Created in 1944 in the Łódź ghetto, it is the only text of this kind in the literature of the Holocaust. Its authors were inmates of the Łódź ghetto, hailing from both the city, as well as from the so-called "Old Reich". They decided to describe the whole community along with its characteristic vocabulary in a form of an encyclopedia. Referring to the introduction by Oskar Rosenfeld dated December 1943, the article presents the idea behind the encyclopedia, its objectives, and its key assumptions. It discusses the structure and construction of entries. Examples have been provided of entries referring to various areas of the ghetto life, with certain entries notably missing from the text, the possible causes of which have been addressed in the article.

Keywords: Ghetto Lodz, archive, Chronicle of the Ghetto, The Encyclopedia of the Ghetto, form and content of the Encyclopedia. 